\title{
Comentário II
}

\author{
Hugues de Varine \\ Ancien directeur du Conseil International des Musées \\ ICOM/UNESCO \\ Consultant en développement local et action \\ communautaire, France
}

Ce texte est un remarquable essai, très fouillé et documenté, de formuler une muséologie critique de l'exposition, présentée justement comme le coeur de tout musée, à la fois sa raison d'être et son mode de communication. La richesse de sa bibliographie, outre qu'elle reflète l'érudition de l'auteur, marque l'extraordinaire diversité et l'accélération de la réflexion et de la recherche sur la muséologie et les disciplines connexes depuis vingt ans environ.

J'en retiens surtouł qu'il est une réponse à une question que je me posais depuis longtemps: quelle est la spécificité de l'exposition par rapport au musée? le musée est essentiellement une institution, réglementée, qui peut répondre à de nombreux besoins et qui est défini, dans divers pays, de manière différente. L'exposition est un moyen d'expression, un média qui peut parfaitemente être autonome, ou bien constituer l'essentiel des programmes d'un musée.

II faudrait d'ailleurs établir une typologie des expositions: permanentes ou temporaires, stables ou itinérantes, professionnelles ou spontanées, liées à un musée ou isolées, etc.. II faudrait ausssi toujours être capable de connaître ou de reconstituer l'objectif ou les objectifs de telle ou telle exposition: la mise en valeur d'une collection, celle d'un chercheur (dans ce cas, c'est souvent le catalogue dit 'raisonné' qui est le vrai but, et non l'expositionl, la vente d'un produit, le complément d'une pédagogie, la démonstration d'une idée, voire d'une idéologie, etc. 
L'article de Ulpiano T.Bezerra de Meneses me semble concerner plutôt, même si ce n'est absolutment pas exclusif, l'exposition comme faisant partie de l'activité permanente d'un musée, dans sa relation à la connaissance historique. Sans vouloir faire ici un examen détaillé de toutes les thèses avancées et discutées, je vais tenter de développer quelques idées qui, tout en étant présentes dans le texte, me paraissent devoir être soulignées comme méritant une attention plus forte et une étude plus approfondie.

L'exposition comme acte politique

Une exposition historique a nécessairement un caractère politique, premier ou au moins secondaire, du moins si elle est autre chose qu'une simple présentation des résultats d'une recherche à l'intention d'autres chercheurs. Car je crois, une fois encore, qu'il faut toujours faire attention à l'objectif: souvent, celui-ci est l'exposition elle-même, car le muséologue a rarement l'esprit politique. Inversement, il peut être ouvertement politique ou idéologique, comme dans le cas das expositions de propagande nationaliste ou vouées à la reconstitution d'une identité culturelle. Mais, généralement, l'objectif est différent, tout en restant implicitement politique: l'exposition prétend par exemple rendre compte d'une problématique, mettre en valeur un ensemble d'objets pour leur donner une signification dans le temps et dans l'espace. Ainsi la présentation d'une culture africaine en Europe, ou d'une découverte archéologique proche-orientale en Amérique, ou d'oeuvres d'art européen à Tokyo pourront apparaître, officiellement, comme une simple manifestation culturelle, elles n'en seront pas moins des reflets et des causes d'interrelations profondes entre des cultures, des peuples, des philoophies, des époques différents. Pire, on assiste à des conflits politiques provoqués par le musée, comme ceux initiés par les trafics internationaux de biens culturels ou la vieille revendication du Bénin en vue de la restitution du trône de son dernier roi, Béhanzin, par le Musée de l'Homme de Paris, où il appartient à une collection dite "ethnographique", alors qu'il est en réalité un simple troophée colonial.

De même, à l'intérieur d'une même société, une exposition historique, ethnographique ou artistique véhiculera des messages "subliminaux" souvent beaucoup plus significatifs que ceux qui ont été voulus par le muséographe ou le chercheur qui lui aura donné son contenu et sa forme. Beaucoup d'expositions sont ainsi au service de l'affirmation de complexes de supériorité, dont la conséquence est à long terme politique, alors que leur but à court terme est ouvertement culturel.

De récents incidents aux Etats-Unis autour, par exemple, de l'exposition commémorative de la bombe d'Hiroshima ont montré que la conscience de ce contenu politique est de plus en plus forte dans les milieux les plus évolués, comme, dans les années 60 , celui des musées de voisinage /neighbourhood museums) aux yeux des militants de l'égalité des races ou des sexes et dans les années 70 celui des musées de sciences naturelles aux yeux des militants de l'écologie. Actuellement, en Inde, de bons observateurs issus du monde des musées traditionnels commencent à se rendre compte de ce que ces institutions, héritées pour l'essentiel de la tradition britannique, fonctionnert comme un outil 
intégrateur au service des cultures dominantes, alors que des milliers de communautés opprimées se voient nier le droit à des cultures spécifiques, autrement que comme des ensembles de spécimens ethnographiques. Et ils recherchent dans la "nouvelle muséologie" ou dans la muséologie communautaire des réponses aux besoins de libération culturelle de ces ethnies.

\section{L'exposition comme processus}

L'exposition apparaît souvent un peu trop comme un "produit" fini, celui qui fait l'objet d'une inauguration et qui va rester ouvert aux consommateurs pendant une période déterminée, peut-être même pour toujours. Il y a naturellement, implicitement, des phases de gestation, de préparation, de construction, de mise en place, qui sont le fait de professionnels. C'est d'ailleurs au coeur de ces phases, dans le non-dit, que Ulpiano de Meneses recherche subtilement des intentions, des mécanismes intellectuels de transformation de l'objet en message. Personnellement, je considère plutôt l'exposition, dans toute sa durée, de l'idée originelle jusqu'à ses suites "politiques", comme un processus non linéaire, vivant, ancré dans l'expérience de son ou de ses concepteurs d'abord, puis dans leur connaissance du sujet, acquise au fur et à mesure de la recherche et de la programmation, enfin dans la relation avec le visiteur.

Ainsi regardée, l'exposition vit sa vie propre, qui peut même, à un certain moment, échapper à son concepteur. Elle peut également vieillir, bien ou mal, si elle se poursuit alors que le contexte dans lequel elle est née change. Notre expérience au Musée de l'Homme et de l'Industrie au Creusot (France) a montré qu'une exposition permanente génialement conçue par G.H.Rivière pouvait, en mois de dix ans, perdre sa pertinence à la suite de bouleversements économiques et sociaux locaux, eux-mêmes reliés à des transformations socio-économiques mondiales. De même, mon expérience de la conception et de la mise au point d'une exposition itinérante sur la vie quotidienne gallo-romaine dans l'Oise /département français au Nord de Paris) m'a montré combien un concept initial simple pouvait devenir complexe et exigeant, une fois prises en compte les caractéristiques du territoire, du public et les demandes de l'archéologie "de sauvetage".

La notion de collection

Je prends résolument parti pour la théorie de ceux qui disent que la collection sur laquelle est basée une exposition (ou un musée) est partout. Sa partie morte se trouve dans le musée, comme des plats cuisinés dans un congélateur: elle est immédiatement disponible, on en connaît les tenants et les aboutissants, elle est à la portée des chercheurs et des étudiants, elle est "conservée". Sa partie vivante est ailleurs et ne cesse pas de se transformer, soit en se détruisant (les biens délaissés et les ensembles archéologiques), soit en s'usant (les objets et documents qui ont une fonction dans la vie), soit en étant thésaurisée (pour des raisons patrimoniales ou affectives). Une exposition ne peut pas se contenter de puiser seulement dans les collections muséalisées, elle ne peut pas ignorer ce qui se trouve "dehors". En 1971, une exposition de trésors 
de l'art yougoslave présentée à Paris a entraîné dans l'année suivante le triplement des vols d'objets d'art dans les églises et les couvents de Yougoslavie... Une exposition sur l'histoire industrielle dans un musée ne peut rendre compte de son sujet sans tenir compte des édifices industriels qui existent sur le terrain, cheminées, usines, maisons, canaux, toutes choses réelles que photos et maquettes ne suffiront jamais à représenter correctement.

Le vrai musée vivant est là, sans conservateurs, sans scénographes ef sans étiquettes, chacun de nous le possède chez lui ou peut le visiter autour de lui. Il y a là d'ailleurs toute une muséologie à inventer, en parallèle et en complémentarité à la muséologie de l'exposition historique: apprendre à regarder ce qui vit peut être aussi "muséologique" que de présenter ce qui est mort.

L'exposition et son public

Une exposition doitelle être vue pour elle-même? Peut-elle exister en dehors d'un public? C'est sans doute là l'une des principales faiblesses de l'article d'Ulpiano de Meneses. Non qu'il oublie le visiteur, le public, mas parce qu'il ne lui accorde pas l'importance qu'il mérite. Pour moi, l'exposition et chacun des objets, documents, ensembles, étiquettes qui la composent n'existent que dans l'intelligence du public. Cela donne nécessairement à l'exposition une multitude de sens, d'interprétations, d'effets, en fonction de ce qui se trouve auparavant, ou même après, dans la mémoire, dans la banque de données cérébrale du visiteur. Je préfère d'ailleurs parler ici du visiteur, être unique dans sa connaissance et dans son expérience, dans son affectivité et dans sa capacité de réaction et de traitement de l'information, plutôt que de public, ou même des publics, expressions qui supposent une uniformité ou des classifications d'ordre statistique: le visiteur 'moyen' n'existe pas.

Toute mon expérience me prouve qu'il existe une relation exclusive entre l'individu et l'objet, puis entre cet individu et le groupe d'objets, enfin entre le même individu et le discours que représente l'exposition. Cette relation est une interaction créative, où l'objet et l'exposition exercent un rôle de catalyse entre deux matières intellectuelles de niveau ef de nature différents: le message émis par la constructin culturelle proposée l'exposition et chacun des objets qu'elle contient), lequel ne sera pas modifié par le contact avec le visiteur, et l'intellect de celui-ci qui, lui, peut être transformé en profondeur, non seulement par la simple addition de nouvelles connaissances, mas surtout par la transformation durable de concepts et de codes, par un apport de créativité.

Pour toutes ces raisons, le visiteur est co-auteur et co-acteur de l'exposition. Il faut le reconnaître comme tel et lui permettre d'avoir, sur la muséologie et sur la muséographie, d'autres idées et d'autres choix que le muséologue professionnel.

Muséologie de l'histoire et le changement

Ma responsabilité professionnelle actuelle est l'action pour le développement et l'association de la communauté à cette action. Le musée ef 
l'exposition en tant que média peuvent et doivent jouer un rôle d'instrument dans les processus de dévelopement, au divers stades de celui-ci. Pour cela, la communauté servie par le musée, qu'elle soit locale, régionale ou nationale, doit être reconnue et associée comme un acteur de l'exposition. C'est à dire que sa culture vivante, comme on l'a vu à propos du public, doit interagir avec le message proposé par l'exposition.

Mais, surtout dans le domaine de la muséologie historique nous devons compter avec un phénomène troublant: I'histoire est affaire de rhytme et de durée, de complexité et de subjectivités. Or l'époque actuelle voit le changement intervenir à grande vitesse, les conditions de vie se modifier substantiellemnt tous les cinq ans environ, le citoyen être appelé à réagir de plus en plus vite à des sollicitations de plus en plus aggressives. L'exposition estelle encore capable de réaliser les conditions de la catalyse décrite plus haut, qui exige de la patience et du temps, de la contemplation, de la réflexion? Comment devons-nous concevoir une muséologie qui contribue effectivement au développemnt et qui facilite la participation du citoyen au développement?

Nous devons, je pense, réinventer une maïeutique adaptée au monde moderne, à mi-chemin entre le Portique athénien et Internet. Le premier était appuyé sur les idées, sur la raison, sur l'abstrait. Le second est appuyé sur la transmission électronique instantanée de mots et d'images. II y a place entre les deux pour l'exposition et pour l'objet, the real thing, un lieu-forum où les questions sont posées, où les subjectivités se heurtent, où les intelligences s'affinent et se reconnaissent. Un historien d'art que j'ai lu dans ma jeunesse, Bernard Berenson, disait quelque part de l'art italien de la Renaissance que ce qui distinguait une école de peinfure d'une autre, c'était la valeur tactile qu'elle recelait ou ne recelait pas. Dans la définition de Berenson, la valeur tactile est ce qui fait penser à un spectateur, après avoir regardé un tableau, qu'il est devenu meilleur. Je pense depuis longtemps qu'une bonne exposition se distingue d'une moins bonne en ce qu'elle a une valeur tactile supérieure.

L'exposition-spectacle: un problème d'éthique

Elle est largement décrite et justement ridiculisée par l'article d'Ulpiano de Meneses. Mais je tiens à y revenir, car elle ne relève pas à mon sens de la muséologie. Nous constatons actuellement dans tous les pays riches une tendance à transformer les grands musées en attractions touristiques intermédiaires entre le parc à thème (style Disneyland) et la galerie commerciale (style Carrefour). La logique commerciale y a pris le pas sur l'objectif culturel, éducatif, et même sur le simple plaisir intellectuel ou visuel. On cultive le visiteur comme unité statistique et source de revenu direct (droits d'entrée et droits "dérivés") ou indirect (effets induits sur le tourisme, les activités commerciales environnantes, la notoriété nationale et internationale.

II serait bon que les muséologues professionnels, comme l'ensemble de la communauté scientifique et culturelle, affirment clairement que ces institutions (les musées) ou ces manifestations (les expositions) ne répondent plus aux définitions et à l'éthique de la muséologie moderne. Elles relèvent de 
l'industrie touristique et de celle du spectacle, tandis que leurs techniques relèvent de la scénographie.

J'ai été frappé, lors de la conférence Générale de l'ICOM à Québec en 1992, par la distance croissante qui sépare le musée-spectacle du musée communautaire. La muséologie de l'exposition historique ne peut être l'esclave des règles du spectacle, ni se lier entièrement aux exigences d'une communauté. Cependant, elle peut et doit apporter sa contribution à l'interprétation dynamique que chacun d'entre nous, comme visiteur et acteur du développement, fera du passé et du présent, à partir d'objets et de témoignages recueillis et présentés qui sont après tout les matériaux avec lesquels nous pouvons construire l'avenir. 